\title{
CONSTRUCTION OF SOME QUANTUM STOCHASTIC OPERATOR COCYCLES BY THE SEMIGROUP METHOD
}

\author{
J. MARTIN LINDSAY AND STEPHEN J. WILLS
}

In celebration of Kalyan Sinha's sixtieth birthday

\begin{abstract}
A new method for the construction of Fock-adapted operator Markovian cocycles is outlined, and its use is illustrated by application to a number of examples arising in physics and probability. The construction uses the Trotter-Kato Theorem and a recent characterisation of such cocycles in terms of an associated family of contraction semigroups.
\end{abstract}

\section{INTRODUCTION}

Let $\mathfrak{h}$ and $\mathbf{k}$ be fixed but arbitrary Hilbert spaces, and let $\mathcal{F}$ denote the symmetric Fock space over $L^{2}\left(\mathbb{R}_{4} ; \mathbf{k}\right)$. In this paper we are concerned with finding left contraction cocycles on $\mathfrak{h}$ with noise dimension space $\mathbf{k}$. That is families $V=\left(V_{t}\right)_{t \geq 0}$ of contractions on $\mathfrak{h} \otimes \mathcal{F}$ that satisfy the functional equation

$$
V_{0}=I, \quad V_{r+t}=V_{r} \sigma_{r}\left(V_{t}\right) \text { for all } r, t \geq 0 .
$$

Here $\left(\sigma_{t}\right)_{t \geq 0}$ is the endomorphism semigroup of right shifts on the algebra $B(\mathfrak{h} \otimes \mathcal{F})$ and the process $V$ is required to be adapted to the natural filtration of subalgebras.

Subject to the further requirement that the cocycle be Markov-regular, there is a one-to-one correspondence between such cocycles and solutions of a class of quantum stochastic differential equations of Hudson-Parthasarathy type. The passage from cocycle to solution of QSDE and back can often still be accomplished without the restrictive Markov-regularity assumption, and so our results also provide a new method for solving QSDEs with unbounded coefficients. In common with earlier work of Fagnola and Mohari ([Fa 3 , Moh] $)$ the resulting cocycle is realised as the limit (in the weak operator topology) of a sequence of Markov-regular cocycles, obtained by Yosida-type regularisation of the coefficients of the QSDE. These authors approximate the cocycle directly, proving the existence of the limit cocycle by appealing to the Arzelà-Ascoli Theorem. This necessitates an assumption of separability on both of the Hilbert spaces $\mathfrak{h}$ and $k$. In contrast, our method goes via an associated family of semigroups and employs the Trotter-Kato Theorem for the approximations. No separability assumptions are needed here; however there are some additional hypotheses. These amount to either assuming relative boundedness of the annihilation coefficients with respect to the time coefficient, or making operator core assumptions on certain affine combinations of annihilation and time coefficients of the QSDE. One goal of this paper is to show that such conditions are natural and easy to check. Both our work and the cited papers of Fagnola and Mohari circumvent limitations of earlier work on the existence of solutions to QSDEs ([Fa, Vin, App]). In order to realise solutions by Picard iteration, as when the coefficients are bounded, those papers imposed strong requirements such as the existence of a dense subspace $\mathfrak{D}$ on $\mathfrak{h}$ that is left invariant by all of the coefficients and on which these operators satisfy suitable growth conditions.

2000 Mathematics Subject Classification. Primary 81S25; Secondary 47D06. 


\section{Cocycles and Semigroups}

In contrast with $\left[\mathrm{LW}_{4}\right]$, which is coordinate-free, our account in this paper will exploit a fixed orthonormal basis $\eta=\left\{d_{i}\right\}_{i \in I}$ of the noise dimension space k. We use an index set $I$ not containing 0 and use $\alpha, \beta$ etc. to denote elements of the augmented set $\widehat{I}:=I \cup\{0\}$, reserving $i$ and $j$ for elements of the set $I$. Let

$$
\begin{gathered}
d_{0}:=0, \quad \mathrm{D}=\operatorname{Lin}\left\{d_{i}\right\}_{i \in I} \subset \mathrm{k} \\
\widehat{\mathrm{k}}:=\mathbb{C} \oplus \mathrm{k}, \quad \widehat{\mathrm{D}}=\mathbb{C} \oplus \mathrm{D}, \quad \widehat{c}=\left(\begin{array}{l}
1 \\
c
\end{array}\right), \\
e_{0}=\widehat{d_{0}}, \quad e_{i}=\left(\begin{array}{l}
0 \\
d
\end{array}\right) \text { where } d=d_{i}, \quad \Delta=I_{\mathfrak{h}} \otimes P_{\mathrm{k}},
\end{gathered}
$$

for $c \in \mathrm{k}$ and $i \in I$, and where $P_{\mathrm{k}} \in B(\widehat{\mathrm{k}})$ is the orthogonal projection with range k. In particular, $\widehat{\eta}:=\left\{e_{\alpha}\right\}_{\alpha \in \widehat{I}}$ is thereby an orthonormal basis of $\hat{k}$ that contains the vector $\left(\begin{array}{l}1 \\ 0\end{array}\right)$ and determines an isomorphism:

$$
\mathfrak{h} \otimes \widehat{\mathbf{k}} \cong \bigoplus_{\alpha \in \widehat{I}} \mathfrak{h} \quad \text { via } \quad \xi \leftrightarrow\left(\xi^{\alpha}\right)_{\alpha \in \widehat{I}} \text { where } \xi^{\alpha}=\left(I_{\mathfrak{h}} \otimes\left\langle e_{\alpha}\right|\right) \xi .
$$

Thus, for any subspace $\mathfrak{D}$ of $\mathfrak{h}$, the algebraic tensor product $\mathfrak{D} \otimes \widehat{\mathbb{D}}$ corresponds to those vectors with only finitely many nonzero components all taken from $\mathfrak{D}$.

Suppose that $\left[F_{\beta}^{\alpha}\right]_{\alpha, \beta \in \widehat{I}}$ is a matrix of operators on $\mathfrak{h}$ and $\mathfrak{D}$ is a subspace of $\mathfrak{h}$ contained in the domain of each $F_{\beta}^{\alpha}$. Then a sesquilinear form is defined on $\mathfrak{D} \underline{\otimes} \widehat{D}$ through

$$
\left(\left(u^{\alpha}\right),\left(v^{\beta}\right)\right) \mapsto \sum_{\alpha, \beta}\left\langle u^{\alpha}, F_{\beta}^{\alpha} v^{\beta}\right\rangle .
$$

Such a matrix can arise as the component matrix of an operator $F$ in $\mathcal{O}(\mathfrak{D} \underline{\otimes} \widehat{D})$, the linear space of operators on $\mathfrak{h} \otimes \widehat{\mathbf{k}}$ with domain $\mathfrak{D} \underline{\otimes} \widehat{\mathbb{D}}$, whose $(\alpha, \beta)$-component is determined by

$$
\left\langle u, F_{\beta}^{\alpha} v\right\rangle=\left\langle u \otimes e_{\alpha}, F v \otimes e_{\beta}\right\rangle, \quad u \in \mathfrak{h}, v \in \mathfrak{D}, \alpha, \beta \in \widehat{I} .
$$

If $\mathrm{k}$ is finite dimensional then clearly all matrices of operators with common dense domain $\mathfrak{D}$ arise in this way. For infinite dimensional $k$ the infinite matrices $\left[F_{\beta}^{\alpha}\right]$ that correspond to an operator on $\mathfrak{D} \underline{\otimes} \widehat{\mathbb{D}}$ are precisely those that satisfy

$$
\sum_{\alpha}\left\|F_{\beta}^{\alpha} u\right\|^{2}<\infty \quad \text { for all } u \in \mathfrak{D}, \beta \in \widehat{I} .
$$

We call such matrices semiregular, extending the terminology introduced in [LW $]$. In the sequel we identify semiregular matrices with their corresponding operators in $\mathcal{O}(\mathfrak{D} \otimes \widehat{\mathrm{D}})$.

Theorem 1.1. Let $\mathfrak{D}$ be a dense subspace of $\mathfrak{h}$. Let $F=\left[F_{\beta}^{\alpha}\right] \in \mathcal{O}(\mathfrak{D} \otimes \underline{\mathbb{D}})$ satisfy

$$
2 \operatorname{Re}\langle\xi, F \xi\rangle+\|\Delta F \xi\|^{2} \leq 0 \quad \text { for all } \xi \in \mathfrak{D} \underline{\otimes} \widehat{\mathrm{D}} \text {. }
$$

Suppose that $F_{0}^{0}$ is the pregenerator of a $C_{0}$-semigroup and either

(i) $F_{i}^{0}$ is relatively bounded with respect to $F_{0}^{0}$ with relative bound 0 for each $i \in I$, or

(ii) $F_{0}^{0}+F_{i}^{0}-\frac{1}{2} I_{\mathfrak{h}}$ is the pregenerator of a $C_{0}$-semigroup for each $i \in I$.

Then the operator QSDE

$$
d V_{t}=\sum_{\alpha, \beta} V_{t} F_{\beta}^{\alpha} d \Lambda_{\beta}^{\alpha}(t), \quad V_{0}=I_{\mathfrak{h} \otimes \mathcal{F}}
$$

has a strong solution $V$ on the domain $\mathfrak{D} \underline{\otimes} \mathcal{E}_{\eta}$. Moreover $V$ is a left contraction cocycle and is the unique contractive weak solution on that domain. 
The following remarks explain the terminology used in the statement of the theorem, and sketch details of the proof, a full account of which can be found in $\left[\mathrm{LW}_{4}\right]$.

1. Solutions of QSDEs: Here $\mathcal{E}_{\eta}$ denotes the linear span of exponential vectors $\varepsilon(f)=\left(1, f,(2 !)^{-1 / 2} f \otimes f, \ldots\right) \in \mathcal{F}$ where $f \in \mathbb{S}_{\eta}$, the set of right continuous step functions on $\mathbb{R}_{+}$, with compact support, taking values in $\eta \cup\{0\}$. This is dense in the Fock space $\mathcal{F}$ ([L], Proposition 2.1).

Three types of QSDE/solution are considered in [LW $\mathrm{LW}_{4}$, namely the form $Q S D E$, and weak or strong solutions of the operator QSDE. The distinction between form QSDE and operator QSDE rests on whether or not the coefficient matrix $\left[F_{\beta}^{\alpha}\right]$ is semiregular, and it can be shown ([LW $\mathrm{LW}_{4}$, Theorem 2.2) that semiregularity of $\left[F_{\beta}^{\alpha}\right]$ is a necessary condition for the existence of contractive solutions of the form version of (1.3). For contraction processes (and the left Hudson-Parthasarathy QSDE considered here), the distinction between weak and strong solutions rests on whether or not the maps $t \mapsto V_{t} \xi$ are weakly or strongly measurable. Our existence result yields a weakly continuous contraction cocycle which is shown to be strongly continuous ([$\left[\mathrm{LW}_{4}\right]$, Lemma $1.2 ;[\mathrm{W}]$, Proposition 1.1), and thus strongly measurable.

Solutions of (1.3) are characterised by their matrix elements through

$$
\begin{aligned}
\left\langle u \otimes \varepsilon(f),\left(V_{t}-I\right) v \otimes \varepsilon(g)\right\rangle & =\int_{0}^{t}\left\langle u \otimes \widehat{f}(s) \otimes \varepsilon(f), \widehat{V}_{s}\left(F \otimes I_{\mathcal{F}}\right) v \otimes \widehat{g}(s) \otimes \varepsilon(g)\right\rangle d s \\
& =\sum_{\alpha, \beta} \int_{0}^{t} \overline{f^{\alpha}(s)} g^{\beta}(s)\left\langle u \otimes \varepsilon(f), V_{s} F_{\beta}^{\alpha} v \otimes \varepsilon(g)\right\rangle d s
\end{aligned}
$$

for $u \in \mathfrak{h}, v \in \mathfrak{D}, f, g, \in \mathbb{S}_{\eta}$, where $\widehat{f}(s):=\widehat{f(s)} \in \widehat{\mathrm{k}}$ is defined through (1.1), $f^{\alpha}(s):=\left\langle e_{\alpha}, f(s)\right\rangle$, a component of $\widehat{f}(s)$ with respect to the basis $\widehat{\eta}$, and $\widehat{V}_{s}$ is $V_{s}$ ampliated to $\mathfrak{h} \otimes \widehat{\mathbf{k}} \otimes \mathcal{F}$.

2. Cocycles and semigroups: There are two key results connecting cocycles to families of semigroups. The first (from $\left[\mathrm{LW}_{2}\right]$ ) uses semigroups to determine which contraction processes are cocycles; the second (from $\left[\mathrm{LW}_{3}\right]$ ) characterises those families of semigroups that can arise in this way, and so can be used to (re) construct a cocycle.

First, let $V=\left(V_{t}\right)_{t \geq 0}$ be an adapted process of contraction operators, that is $V_{t} \in B\left(\mathfrak{h} \otimes \mathcal{F}_{[0, t]}\right) \otimes I_{[t, \infty[}$ where $\mathcal{F}_{J}$ denotes the symmetric Fock space over $L^{2}(J ; \mathrm{k})$ and $I_{J}$ is the identity operator on $\mathcal{F}_{J}$. For any $c, d \in \mathrm{k}$ define contractions $Q_{t}^{c, d} \in B(\mathfrak{h})$ through

$$
\left\langle u, Q_{t}^{c, d} v\right\rangle=\left\langle u \otimes \varpi\left(c \mathbf{1}_{[0, t[}\right), V_{t} v \otimes \varpi\left(d \mathbf{1}_{[0, t[}\right)\right\rangle,
$$

where $\varpi(f)=\|\varepsilon(f)\|^{-1} \varepsilon(f)$, the normalised exponential vector associated to $f$. From [LW $\mathrm{LW}_{2}$ it follows that the process $V$ is a left contraction cocycle if and only if $\left(Q_{t}^{c, d}\right)_{t \geq 0}$ is a semigroup on $\mathfrak{h}$ for each pair $c, d \in \mathbf{k}$, and

$$
\left\langle u \otimes \varpi\left(f \mathbf{1}_{[0, t[}\right), V_{t} v \otimes \varpi\left(g \mathbf{1}_{[0, t]}\right)\right\rangle=\left\langle u, Q_{t_{1}-t_{0}}^{f\left(t_{0}\right), g\left(t_{0}\right)} \cdots Q_{t-t_{n}}^{f\left(t_{n}\right), g\left(t_{n}\right)} v\right\rangle
$$

for all $u, v \in \mathfrak{h}$ and $f, g \in \mathbb{S}_{\eta}$, where $\left\{0=t_{0} \leq t_{1} \leq \cdots \leq t_{n} \leq t\right\}$ contains the discontinuities of $f \mathbf{1}_{[0, t[}$ and $g \mathbf{1}_{[0, t[}$.

This characterisation is independent of continuity assumptions in $t$. However weak continuity of the map $t \mapsto V_{t}$ is equivalent to strong continuity, which is equivalent to strong continuity of all (equivalently any) of the semigroups $Q^{c, d}$. In particular if we assume this continuity and set $Q^{(\alpha, \beta)}=Q^{c, d}$ for $c=d_{\alpha}, d=d_{\beta}$, then each of these $C_{0}$-semigroups has a generator $G_{(\alpha, \beta)}$. If $\mathfrak{D}:=\bigcap_{\alpha, \beta} \operatorname{Dom} G_{(\alpha, \beta)}$ 
is a dense subspace of $\mathfrak{h}$ then it is not hard to show that $V$ satisfies the QSDE (1.3) on $\mathfrak{D} \underline{\otimes} \mathcal{E}_{\eta}$ where the $F_{\beta}^{\alpha}$ are given by

$$
\begin{aligned}
F_{0}^{0} & =G_{(0,0)}, \\
F_{0}^{i} & =G_{(i, 0)}-G_{(0,0)}+\frac{1}{2} I_{\mathfrak{h}}, \quad F_{j}^{0}=G_{(0, j)}-G_{(0,0)}+\frac{1}{2} I_{\mathfrak{h}}, \\
F_{j}^{i} & =G_{(i, j)}-G_{(i, 0)}-G_{(0, j)}+G_{(0,0)}-\delta_{j}^{i}
\end{aligned}
$$

for $i, j \in I$, and where $\delta_{j}^{i}$ is the Kronecker delta. This is Theorem 4.1 of [ $\left[\mathrm{LW}_{4}\right]$, proved there using a more direct method for extracting the $F_{\beta}^{\alpha}$. It extends Theorem 6.7 of $\left[\mathrm{LW}_{2}\right]$ when one, and hence all, of the semigroups is assumed to be norm continuous.

Thus associated to any cocycle is a family of semigroups. Conversely, the following characterisation is obtained in $\left[\mathrm{LW}_{3}\right]$. A family $\mathcal{Q}=\left\{Q^{c, d}: c, d \in \mathrm{D}\right\}$ of semigroups on $\mathfrak{h}$ is amongst those associated to a left contraction cocycle through (1.4) if and only if for all $n \in \mathbb{N}, Y \in \mathrm{M}_{n}(|\mathfrak{h}\rangle)$ and positive invertible matrices $A, B \in$ $\mathrm{M}_{n}(\mathbb{C})$,

$$
\begin{aligned}
& \left\|A^{-1 / 2} Y B^{-1 / 2}\right\| \leq 1 \text { implies } \\
& \quad\left\|\left(A \bullet \varpi_{t}^{\mathbf{c}}\right)^{-1 / 2}\left(Q_{t}^{\mathbf{c}} \bullet Y\right)\left(B \bullet \varpi_{t}^{\mathbf{c}}\right)^{-1 / 2}\right\| \leq 1 \text { for all } \mathbf{c} \in \mathrm{D}^{n}, t \geq 0 .
\end{aligned}
$$

Here $|\mathfrak{h}\rangle=B(\mathbb{C} ; \mathfrak{h})$, the column operator space associated to $\mathfrak{h}$ (so that $\mathrm{M}_{n}(|\mathfrak{h}\rangle$ ) is identified with $\left.B\left(\mathbb{C}^{n} ; \mathfrak{h}^{n}\right)\right), \varpi_{t}^{\mathbf{c}}$ is the scalar matrix $\left[\left\langle\varpi\left(c_{i} \mathbf{1}_{[0, t[}\right), \varpi\left(c_{j} \mathbf{1}_{[0, t]}\right)\right\rangle\right] \in$ $\mathrm{M}_{n}(\mathbb{C}), Q_{t}^{\mathbf{c}}$ is the operator matrix $\left[Q_{t}^{c_{i}, c_{j}}\right] \in B\left(\mathfrak{h}^{n}\right)$, and $\bullet$ denotes the Schur product.

The usefulness of this result may be explained as follows. Suppose that $\left(V^{(n)}\right)_{n \geq 1}$ is a sequence of cocycles whose associated semigroups converge in the strong operator topology to a new family $\mathcal{Q}=\left\{Q^{c, d}\right\}$ of semigroups $\left({ }^{(n)} Q_{t}^{c, d} u \rightarrow Q_{t}^{c, d} u\right.$ for all $c, d \in \mathrm{D}, t \geq 0, u \in \mathfrak{h})$. Then, since (1.7) is manifestly stable under pointwise limits, there must be a left contraction cocycle whose associated semigroups include $\mathcal{Q}$.

3. Trotter-Kato Theorem for cocycles/Proof of Theorem 1.1: Requiring contractivity of solutions of (1.3) imposes further useful necessary conditions on the putative 'stochastic generator' $F=\left[F_{\beta}^{\alpha}\right] \in \mathcal{O}(\mathfrak{D} \otimes \mathrm{D})$, namely:

- $F$ satisfies the form inequality (1.2);

- each $F_{0}^{i}$ is relatively bounded with respect to $F_{0}^{0}$ with relative bound 0 ;

- each $G_{(\alpha, \beta)}$ defined through (1.6) is dissipative;

- $\left[F_{j}^{i}+\delta_{j}^{i} I\right]_{i, j \in I}$ defines a contraction on $\mathfrak{h} \otimes \mathbf{k}$;

- If $F_{0}^{0}$ is a pregenerator of a $C_{0}$-contraction semigroup then there is a sequence $\left(F^{(n)}\right)_{n \geq 1}$ in $B(\mathfrak{h} \otimes \widehat{\mathbf{k}})$ with each $F^{(n)}$ satisfying (1.2), and such that $F^{(n)} \rightarrow F$ strongly on $\mathfrak{D} \underline{\otimes}$.

Most of these are noted in $\left[\mathrm{Fa}_{3}\right]$; they are all contained in Propositions 2.1, 3.1 and 3.2 of $\left[\mathrm{LW}_{4}\right]$. For each $n$, the QSDE (1.3) with coefficient $F^{(n)}$ can be solved by Picard iteration, and since $F^{(n)}$ satisfies (1.2) the solution $V^{(n)}$ is a Markov-regular left contraction cocycle. Thus each $V^{(n)}$ has an associated family of semigroups $\mathcal{Q}^{(n)}=\left\{{ }^{(n)} Q^{(\alpha, \beta)}\right\}$, whose corresponding generators $G_{(\alpha, \beta)}^{(n)}$ are affine combinations of the components of $F^{(n)}$ (as in (1.6)), and so converge strongly to $G_{(\alpha, \beta)}$ on $\mathfrak{D}$. In turn these are pregenerators of $C_{0}$-contraction semigroups - this is deduced from a combination of the necessary conditions on $F$ listed above and either condition (i) or (ii) of Theorem 1.1. The resulting family $\mathcal{Q}$ satisfies (1.7), and so we obtain the desired left contraction cocycle $V$; moreover $V_{t}^{(n)} \rightarrow V_{t}$ for each $t \geq 0$ in the weak operator topology. 
A different type of semigroup appears in the characterisation of the coefficients $F$ which yield isometric solutions for the QSDE (1.3). If $F \in B(\mathfrak{h} \otimes \widehat{\mathbf{k}})$ then $V$ is isometric if and only if $F+F^{*}+F^{*} \Delta F=0$, that is, equality holds in (1.2) $\left(\left[\mathrm{Fa}_{3}, \mathrm{LW}_{1}\right]\right)$. For unbounded generators this condition is still necessary, but it is no longer sufficient. If $V=\left(V_{t}\right)_{t \geq 0}$ is a contractive solution of (1.3), then defining

$$
\mathcal{T}_{t}(X):=\mathbb{E}\left[V_{t}^{*}\left(X \otimes I_{\mathcal{F}}\right) V_{t}\right], \quad X \in B(\mathfrak{h}),
$$

where $\mathbb{E}$ is the vacuum conditional expectation $B(\mathfrak{h} \otimes \mathcal{F}) \rightarrow B(\mathfrak{h})$, produces a family of normal completely positive contractions on $B(\mathfrak{h})$. Moreover $\left(\mathcal{T}_{t}\right)_{t>0}$ is a quantum dynamical semigroup, so that $\mathcal{T}_{0}=\operatorname{id}_{B(\mathfrak{h})}, \mathcal{T}_{r+t}=\mathcal{T}_{r} \circ \mathcal{T}_{t}$, and $t \mapsto \mathcal{T}_{t}(X)$ is ultraweakly continuous. It is the minimal quantum dynamical semigroup with form-generator $\mathcal{L}$, where

$$
\mathcal{L}(X):(u, v) \mapsto\left\langle u, X F_{0}^{0} v\right\rangle+\left\langle F_{0}^{0} u, X v\right\rangle+\sum_{i}\left\langle F_{0}^{i} u, X F_{0}^{i} v\right\rangle, \quad u, v \in \mathfrak{D},
$$

meaning that

$$
\left\langle u,\left(\mathcal{T}_{t}(X)-X\right) v\right\rangle=\int_{0}^{t} \mathcal{L}\left(\mathcal{T}_{s}(X)\right)(u, v) d s
$$

and

$$
\mathcal{T}_{t}(X) \leq \mathcal{T}_{t}^{\prime}(X) \text { for all } t \geq 0 \text { and } X \in B(\mathfrak{h})_{+}
$$

for any other quantum dynamical semigroup $\mathcal{T}^{\prime}$ that satisfies (1.9). See [Fa $]$ for more details.

Theorem $1.2\left(\left[\mathrm{Fa}_{3}, \mathrm{Fa}_{4}\right]\right)$. Suppose that $F \in \mathcal{O}(\mathfrak{D} \underline{\otimes} \mathrm{D})$ satisfies

$$
2 \operatorname{Re}\langle\xi, F \xi\rangle+\|\Delta F \xi\|^{2}=0 \quad \text { for all } \xi \in \mathfrak{D} \underline{\otimes} \mathrm{D}
$$

and that $F_{0}^{0}$ is a pregenerator of a $C_{0}$-contraction semigroup. Suppose also that there is a contractive solution $V$ to (1.3) for this $F$, and define $\mathcal{T}$ by (1.8). Then $V$ is isometric if and only if $\mathcal{T}$ is conservative, that is, $\mathcal{T}_{t}(I)=I$ for all $t \geq 0$.

A number of necessary and sufficient conditions have been found for conservativity of the quantum dynamical semigroup $\mathcal{T}$; however in practice these can be difficult to verify, so a number of sufficient conditions have been developed (see [ChF] and references therein).

To determine if $V$ is coisometric involves analysing its Journé dual. Taking adjoints exchanges left cocycles and right cocycles (satisfying $U_{r+t}=\sigma_{r}\left(U_{t}\right) U_{r}$ ); similarly conjugating by time-reversal operators converts a left cocycle into a right cocycle and vice versa. Thus conjugating the adjoint of a left cocycle $V$ yields another left cocycle $\widetilde{V}$, and if $V$ satisfies (1.3) for $F=\left[F_{\beta}^{\alpha}\right]$ then, at least formally, $\widetilde{V}$ should satisfy (1.3) for the adjoint matrix $F^{\dagger}:=\left[F_{\alpha}^{\beta^{*}}\right]$, so that one can seek to apply Theorem 1.2 to this cocycle. This has been carried out successfully in many instances $\left(\left[\mathrm{Fa}_{4}\right]\right)$.

\section{EXAMPLES}

2.1. A nonisometric cocycle as a limit of isometric cocycles. Let $\mathfrak{h}=l^{2}\left(\mathbb{Z}_{+}\right)$ with its standard orthonormal basis $\left(e_{n}\right)_{n \geq 0}$, let $W \in B(\mathfrak{h})$ be the isometric right shift $W e_{n}=e_{n+1}$, and let $L$ be its Cayley transform: $i(I+W)(I-W)^{-1}$. Thus $L$ is closed, densely defined and symmetric, but not self-adjoint. In view of the identity $\operatorname{Dom} L^{*}=\operatorname{Dom} L+\mathbb{C e}_{0}$, if we set $\mathfrak{D}=\operatorname{Dom} L^{*} L$ and $\mathrm{k}=\mathbb{C}$, then

$$
\left[\begin{array}{cc}
-\frac{1}{2} L^{*} L & -L^{*} \\
L & 0
\end{array}\right]
$$

restricts to an operator $F \in \mathcal{O}(\mathfrak{D} \oplus \mathfrak{D})$, and moreover $F$ satisfies (1.10). Since $L^{*} L$ is a positive self-adjoint operator, $F_{0}^{0}$ is the generator of a $C_{0}$-contraction semigroup. 
Also, since $-L^{*} \supset-L, F_{1}^{0}$ is relatively bounded with respect to $F_{0}^{0}$ with relative bound 0 . Thus condition (i) of Theorem 1.1 applies. Let $V$ be the solution to (1.3) for this $F$.

The related quantum dynamical semigroup $\mathcal{T}$ is not conservative, as noted in Example 3.5 of [BhS], hence $V$ cannot be isometric by Theorem 1.2. However, $V$ is constructed by regularising $F$ to produce a sequence $\left(F^{(n)}\right)_{n \geq 1}$ with $F^{(n)} \rightarrow F$ strongly, so that for the semigroup generators $G_{(\alpha, \beta)}^{(n)} \rightarrow G_{(\alpha, \beta)}$ strongly on $\mathfrak{D}$, hence ${ }^{(n)} Q_{t}^{(\alpha, \beta)} \rightarrow Q_{t}^{(\alpha, \beta)}$ strongly as well, by the Trotter-Kato Theorem ([EnN], Theorem III.4.8). Now for each $n \in \mathbb{N}, F^{(n)}$ lies in $B(\mathfrak{h} \oplus \mathfrak{h})$ and satisfies (1.10), hence each cocycle $V^{(n)}$ is isometric. Thus, although (1.5) readily guarantees the weak operator convergence $V_{t}^{(n)} \rightarrow V_{t}$ in our stochastic generalisation of the TrotterKato Theorem, this example shows that convergence of the cocycles in the strong operator topology cannot be guaranteed.

Technical interlude. Let $\mathfrak{h}=l^{2}(\mathbb{Z})$, and let $W$ be the right shift operator. Given functions $\lambda, \mu: \mathbb{Z} \rightarrow \mathbb{C}$ we will consider operators such as $\lambda(N)+W \mu(N)$ and $\lambda(N)+$ $\mu(N) W^{*}$, where $N$ denotes the number operator. If $\left(e_{n}\right)_{n \in \mathbb{Z}}$ denotes the standard orthonormal basis of $\mathfrak{h}$ then these operators leave $\mathfrak{D}:=\operatorname{Lin}\left\{e_{n}\right\}$ invariant and their action is given there by $(\lambda(N)+W \mu(N)) e_{n}=\lambda(n) e_{n}+\mu(n) e_{n+1}$. However, despite the temptation to restrict everything to $\mathfrak{D}$ and thus simplify various analytical domain considerations, we shall also have need to think of these operators acting on their maximal domains. For example: $\lambda(N)+W \mu(N)$ has maximal domain

$$
\left\{u=\left(u_{n}\right) \in \mathfrak{h}: \sum_{n}\left|\lambda(n) u_{n}+\mu(n-1) u_{n-1}\right|^{2}<\infty\right\} .
$$

Let $\lambda(N) \dot{+} W \mu(N)$ denote the resulting operator. An advantage of passing to these domains is that we then have the true operator equalities

$$
\left(\lambda(N)+\left.W \mu(N)\right|_{\mathfrak{D}}\right)^{*}=(\lambda(N) \dot{+} W \mu(N))^{*}=\bar{\lambda}(N) \dot{+} \bar{\mu}(N) W^{*},
$$

the subspace $\mathfrak{D}$ being a core for such operators.

Remark. In general the maximal domain is strictly larger than that of the operator sum, namely $\operatorname{Dom} \lambda(N) \cap \operatorname{Dom} \mu(N)$. Indeed, if we take $\lambda(n)=n, \mu(n)=-n$ and set $u=\left(u_{n}\right)$ where $u_{n}=(1+|n|)^{-1}$ then $u \in \mathfrak{h}, u \notin \operatorname{Dom} \lambda(N)=\operatorname{Dom} \mu(N)$, but

$$
\sum_{n \in \mathbb{Z}}\left|\lambda(n) u_{n}+\mu(n-1) u_{n-1}\right|^{2}=\sum_{n<0}[(1-n)(2-n)]^{-2}+\frac{1}{4}+\sum_{n>0}[n(n+1)]^{-2}<\infty,
$$

so that $u$ lies in the maximal domain of $\lambda(N)+W \mu(N)$.

2.2. The inverse harmonic oscillator. Let $\mathfrak{h}=l^{2}\left(\mathbb{Z}_{+}\right)$with its standard orthonormal basis $\left(e_{n}\right)_{n \geq 0}$, let $\mathfrak{D}=\operatorname{Lin}\left\{e_{n}\right\}$, and again let $W$ be the isometric right shift on $\mathfrak{h}$. Define $F \in \mathcal{O}(\mathfrak{D} \oplus \mathfrak{D})$ by

$$
F=\left[\begin{array}{cc}
-\frac{1}{2}|\lambda|^{2}(N+1)+i \mu(N) & W^{*} \bar{\lambda}(N) \\
-\lambda(N) W & 0
\end{array}\right],
$$

where $\lambda: \mathbb{Z}_{+} \rightarrow \mathbb{C}$ and $\mu: \mathbb{Z}_{+} \rightarrow \mathbb{R}$ are arbitrary functions. The components of $F$, viewed as operators on $\mathfrak{D}$, each leave $\mathfrak{D}$ invariant and it is not hard to verify that $F$ satisfies (1.10).

Now $F_{0}^{0} \in \mathcal{O}(\mathfrak{D})$ is dissipative, and $\left(\nu-F_{0}^{0}\right)(\mathfrak{D})=\mathfrak{D}$ for each $\nu>0$, hence $\overline{F_{0}^{0}}$ is the generator of a $C_{0}$-contraction semigroup. If there is $c>0$ such that

$$
c|\lambda(n)| \leq|\lambda(n+1)| \quad \text { for all } n \geq 0
$$


then, for each $u \in \mathfrak{D}$ and $a>0$, we have

$$
\left\|F_{1}^{0} u\right\|^{2} \leq\left\langle u,|\lambda|^{2}(N) u\right\rangle \leq \frac{a^{2}}{2}\|u\|^{2}+\frac{1}{2 a^{2}}\|\lambda(N) u\|^{2} \leq \frac{a^{2}}{2}\|u\|^{2}+\frac{2}{a^{2} c^{4}}\left\|F_{0}^{0} u\right\|^{2},
$$

and so condition (i) of the theorem is satisfied. A particular case where (2.2) holds is if we take $\lambda(n)=\sqrt{n}$; this was considered in [Wal]. However examples where condition (i) of the theorem is violated are easily generated. For example, setting

$$
\mu=0 \text { and } \lambda(n)= \begin{cases}\sqrt{n} & \text { if } n \text { is odd, } \\ 0 & \text { if } n \text { is even, }\end{cases}
$$

then $\left\|F_{1}^{0} e_{2 m+1}\right\|=\sqrt{2 m+1}$ but $\left\|F_{0}^{0} e_{2 m+1}\right\|=0$ for each $m \geq 0$.

In fact every $F$ of the form (2.1) stochastically generates a cocycle since condition (ii) of Theorem 1.1 always holds. This can be seen using the following special case of a well-known result ([EnN], Corollary II.3.17), whose short proof we include for the convenience of the reader.

Lemma 2.1. Let $A$ be a dissipative operator with dense domain $\mathfrak{D}$. If $\mathfrak{D} \subset$ Dom $A^{*}$ and $\mathfrak{D}$ is core for $A^{*}$ then $A$ is a pregenerator of a $C_{0}$-contraction semigroup.

Proof. By the Lumer-Phillips Theorem it suffices to show that $I-A$ has dense range. Let $v \in \operatorname{Ran}(I-A)^{\perp}$, then

$$
\langle(I-A) u, v\rangle=0 \text { for all } u \in \mathfrak{D} \Rightarrow v \in \operatorname{Dom} A^{*} \text { with }\left(I-A^{*}\right) v=0 .
$$

Dissipitavity of $A$ and the core assumption imply that $A^{*}$ is also dissipative, so

$$
\left\|\left(I-A^{*}\right) v\right\|\|v\| \geq \operatorname{Re}\left\langle\left(I-A^{*}\right) v, v\right\rangle \geq\|v\|^{2},
$$

hence $v=0$ as required.

The putative stochastic generator $F$ above satisfies the equality (1.10). It follows that the operator

$$
A:=F_{0}^{0}+F_{1}^{0}-\left.\frac{1}{2} I\right|_{\mathfrak{D}}
$$

is dissipative. Moreover $\mathfrak{D}$ is a core for $A^{*}=\frac{1}{2}|\lambda|^{2}(N+1) \dot{+} i \mu(N) \dot{-} W^{*} \bar{\lambda}(N) \dot{-} \frac{1}{2} I$, from the technical remarks above, and so Lemma 2.1 applies. Thus condition (ii) of Theorem 1.1 holds.

2.3. Birth and death processes. Now let $\mathfrak{h}=l^{2}(\mathbb{Z})$, again write the standard orthonormal basis as $\left(e_{n}\right)_{n \in \mathbb{Z}}$, set $\mathfrak{D}=\operatorname{Lin}\left\{e_{n}\right\}$ and let $W$ be the unitary right shift. This time let $\mathrm{k}=\mathbb{C}^{2}$ and let $F \in \mathcal{O}\left(\mathfrak{D} \underline{\otimes} \mathbb{C}^{3}\right)$ be given by

$$
F=\left[\begin{array}{ccc}
-\frac{1}{2}|\lambda|^{2}(N)-\frac{1}{2}|\mu|^{2}(N) & \bar{\lambda}(N) W^{*} & \bar{\mu}(N) W \\
-\lambda(N) & W^{*}-I & 0 \\
-\mu(N) & 0 & W-I
\end{array}\right]
$$

where $\lambda, \mu: \mathbb{Z} \rightarrow \mathbb{C}$ are arbitrary functions. Again the structure of $F$ and the fact that each component of $F$ leaves $\mathfrak{D}$ invariant both facilitate the verification of (1.10). Careful choice of $\mu$ and $\lambda$ again shows that condition (i) of Theorem 1.1 need not hold. However Lemma 2.1 and the technical remarks once more imply that $F_{0}^{0}+F_{1}^{0}-\left.\frac{1}{2} I\right|_{\mathfrak{D}}$ and $F_{0}^{0}+F_{2}^{0}-\left.\frac{1}{2} I\right|_{\mathfrak{D}}$ are indeed pregenerators of $C_{0}$-semigroups, so that condition (ii) of Theorem 1.1 is satisfied.

Remark. In general it is not always possible to apply Theorem 1.2 (to $F$ and its adjoint) to conclude that $V$ is unitary. The functions $\lambda$ and $\mu$ that lead to unitary solutions have been studied in detail in $\left[\mathrm{Fa}_{2}\right]$. Necessary growth conditions on $\lambda$ and $\mu$ for unitarity of $V$ are obtained from a reformulation of the well-known resolvent conditions of Feller for the Kolmogorov equations in the classical theory of Markov processes - see [MoP] for further remarks in this vein. 
2.4. A system for second harmonic generation. In [GiP] an example is given involving two oscillators coupled by a nonlinear interaction term. To obtain a quantum stochastic dilation take $\mathfrak{h}=l^{2}\left(\mathbb{Z}_{+}^{2}\right)=l^{2}\left(\mathbb{Z}_{+}\right) \otimes l^{2}\left(\mathbb{Z}_{+}\right)$with orthonormal basis $\left\{e_{m, n}=e_{m} \otimes e_{n}\right\}_{m, n \in \mathbb{Z}_{+}}$, and define creation and annihilation operators on each copy of $l^{2}\left(\mathbb{Z}_{+}\right)$, so that, for example,

$$
a_{1}^{*} e_{m, n}=\sqrt{m+1} e_{m+1, n}, \quad a_{2} e_{m, n}= \begin{cases}0 & \text { if } n=0 \\ \sqrt{n} e_{m, n-1} & \text { if } n \geq 1 .\end{cases}
$$

Let $\mathfrak{D}=\operatorname{Lin}\left\{e_{m, n}\right\}, \mathrm{k}=\mathbb{C}^{2}$ and define $F \in \mathcal{O}\left(\mathfrak{D} \otimes \mathbb{C}^{3}\right)$ by

$$
F=\left[\begin{array}{ccc}
K & -a_{1}^{*} & -a_{2}^{*} \\
a_{1} & 0 & 0 \\
a_{2} & 0 & 0
\end{array}\right]
$$

where

$$
K=\frac{1}{2}\left(a_{1}^{*} a_{1}+a_{2}^{*} a_{2}\right)+\omega\left(a_{1}^{*}-a_{1}\right)+\lambda\left(a_{1}^{* 2} a_{2}-a_{1}^{2} a_{2}^{*}\right)
$$

for some $\omega, \lambda \in \mathbb{R}$. Now clearly $F$ leaves $\mathfrak{D} \otimes \mathbb{C}^{3}$ invariant, and it is easy to verify that $F$ satisfies (1.10). In particular $K \in \mathcal{O}(\mathfrak{D})$ is dissipative, $\mathfrak{D}$ is a core for $K^{*}$, and so $\bar{K}$ is the generator of a contraction semigroup by Lemma 2.1. By the same reasoning so are $\overline{K-a_{1}^{*}-\frac{1}{2} I}$ and $\overline{K-a_{2}^{*}-\frac{1}{2} I}$. Hence condition (ii) of Theorem 1.1 holds, and so $F$ generates a contraction cocycle $V$.

Remarks. (i) A more detailed analysis of $K$, or rather of $K-\omega\left(a_{1}^{*}-a_{1}\right)$, as has been done by Fagnola $\left(\left[\mathrm{Fa}_{5}\right]\right)$ using the results of $[\mathrm{ChF}]$, shows that the associated quantum dynamical semigroup is conservative, and hence that the cocycle $V$ is isometric by Theorem 1.2. Using the results of [FaR], Fagnola has also established the existence of an invariant state for the semigroup.

(ii) The stochastic generator $F$ may be perturbed by introducing nonzero number/exchange coefficients, without changing the quantum dynamical semigroup, or complicating the application of Theorem 1.1, provided that the annihilation coefficients are appropriately modified. For example, the above argument works equally well if we take

$$
F=\left[\begin{array}{ccc}
K & -a_{1}^{*} V_{1} & -a_{2}^{*} V_{2} \\
a_{1} & V_{1}-I & 0 \\
a_{2} & 0 & V_{2}-I
\end{array}\right]
$$

for unitary operators $V_{1}$ and $V_{2}$ which leave $\mathrm{D}$ invariant, for example operators permuting the basis vectors $\left\{e_{m, n}\right\}$.

ACKNOWLEDGEMENTS. We are grateful to Franco Fagnola for drawing our attention to $[\mathrm{GiP}]$, and sharing his results in this area. SJW acknowledges support from EU TMR Networks HPRN-CT-2002-00279 and HPRN-CT-2002-00280.

\section{REFERENCES}

[App] D. Applebaum, Unitary evolutions and horizontal lifts in quantum stochastic calculus, Comm. Math. Phys. 140 (1991), 63-80.

[BhS] B.V. Rajarama Bhat and K.B. Sinha, Examples of unbounded generators leading to nonconservative minimal semigroups, in "Quantum Probability and Related Topics, QP-PQ IX" ed. L. Accardi, World Scientific, Singapore 1994, pp.89-103.

[ChF] A.M. Chebotarev and F. Fagnola, Sufficient conditions for conservativity of minimal quantum dynamical semigroups, J. Funct. Anal. 153 (1998), 382-404.

[EnN] K.-J. Engel and R. Nagel, "One-parameter semigroups for linear evolution equations," Graduate Texts in Mathematics 194, Springer-Verlag, New York, 2000.

[Fa $\left.{ }_{1}\right]$ F. Fagnola, On quantum stochastic differential equations with unbounded coefficients, Probab. Theory Related Fields 86 (1990), 501-516.

$\left[\mathrm{Fa}_{2}\right]$ F. Fagnola, Pure birth and death processes as quantum flows in Fock space, Sankhya Ser. A 53 (1991), 288-297. 
$\left[\mathrm{Fa}_{3}\right]$ F. Fagnola, Characterization of isometric and unitary weakly differentiable cocycles in Fock space, in "Quantum Probability and Related Topics, QP-PQ VIII," ed. L. Accardi, World Scientific, Singapore 1993, pp.143-164.

[Fa4 4 F. Fagnola, Quantum Markov semigroups and quantum flows, Proyecciones 18 (1999).

$\left[\mathrm{Fa}_{5}\right]$ F. Fagnola, private communication, 2003.

[FaR] F. Fagnola and R. Rebolledo, On the existence of stationary states for quantum dynamical semigroups, J. Math. Phys. 42 (2001), 1296-1308.

[FaW] F. Fagnola and S.J. Wills, Solving quantum stochastic differential equations with unbounded coefficients, J. Funct. Anal. 198 (2003), 279-310.

[GiP] N. Gisin and I.C. Percival, The quantum-state diffusion model applied to open systems, $J$. Phys. A 25 (1992), 5677-5691.

[L] J.M. Lindsay, Quantum stochastic analysis - an introduction, in D. Applebaum, B.V.R. Bhat, J. Kustermans and J.M. Lindsay, "Quantum Independent Increment Processes I: from Classical Probability to Quantum Stochastic Calculus," eds. U. Franz $\mathcal{E}$ M. Schürmann, Lecture Notes in Mathematics 1865, Springer, Heidelberg 2005.

$\left[\mathrm{LW}_{1}\right]$ J.M. Lindsay and S.J. Wills, Existence, positivity, and contractivity for quantum stochastic flows with infinite dimensional noise, Probab. Theory Related Fields 116 (2000), 505-543.

$\left[\mathrm{LW}_{2}\right]$ J.M. Lindsay and S.J. Wills, Markovian cocycles on operator algebras, adapted to a Fock filtration, J. Funct. Anal. 178 (2000), 269-305.

$\left[\mathrm{LW}_{3}\right]$ J.M. Lindsay and S.J. Wills, Quantum stochastic cocycles and completely bounded semigroups on operator spaces I, preprint.

[LW 4 J.M. Lindsay and S.J. Wills, Quantum stochastic operator cocycles via associated semigroups, Math. Proc. Cambridge Philos. Soc. (to appear). arXiv:math.FA/0512398.

[Moh] A. Mohari, Quantum stochastic differential equations with unbounded coefficients and dilations of Feller's minimal solution, Sankhyā Ser. A 53 (1991), 255-287.

[MoP] A. Mohari and K.R. Parthasarathy, A quantum probabilistic analogue of Feller's condition for the existence of unitary Markovian cocycles in Fock spaces, in "Statistics and Probability: A Raghu Raj Bahadur Festschrift," eds. J.K. Ghosh, S.K. Mitra, K.R. Parthasarathy \& B.L.S. Prakasa Rao, Wiley Eastern, New Delhi 1993, pp.475-497.

[Vin] G.F. Vincent-Smith, Unitary quantum stochastic evolutions, Proc. London Math. Soc. 63 (1991), 401-425.

[Wal] W. von Waldenfels, Continuous Maassen kernels and the inverse oscillator, in "Séminaire de Probabilités XXX," eds. J. Azéma, M. Emery \& M. Yor, Lecture Notes in Mathematics 1626, Springer, Heidelberg 1996, pp.117-161.

[W] S.J. Wills, On the generators of quantum stochastic operator cocycles, Markov Proc. Related Fields (to appear). arXiv:math-ph/0510040.

Department of Mathematics and Statistics, Lancaster University, Lancaster LA1 $4 \mathrm{YF}, \mathrm{UK}$

E-mail address: j.m.lindsay@lancaster.ac.uk

School of Mathematical Sciences, University College Cork, Cork, Ireland

E-mail address: s.wills@ucc.ie 